\title{
NON-RADIAL PULSATIONS OF DWARF NOVAE
}

\author{
BRIAN WARNER \\ University of Cape Town, Rondebosch, South Africa
}

\begin{abstract}
The high speed photometric observations of dwarf novae during outbursts that led to the discovery (Warner and Robinson, 1972) of rapid pulsations have been extended to other objects. Pulsations are usually seen near outburst maximum; an exception is VW Hyi which showed oscillations on December 25, 1972, when it was well down the declining part of its light curve.

The periods of the oscillations are found to vary; in most cases a rapid change from one discrete mode to another is observed. The dwarf nova stays in one oscillation mode for about an hour, and changes mode over a time of about $10 \mathrm{~min}$. In the case of the VW Hyi observation, the period changed continuously from $28 \mathrm{~s}$ to $34 \mathrm{~s}$ over a time of $4 \mathrm{~h}$, during which the apparent brightness decreased by $40 \%$.

The existence of many closely spaced oscillation modes suggests that the dwarf novae are oscillating in non-radial modes. This is supported by observations of the 71-s oscillations of DQ Her: during eclipse a phase shift is seen indicating an $l=2$, $m=2$ mode of oscillation. In UX UMa, the 29-s oscillations show an opposite phase shift from that in DQ Her and indicate an $l=2, m=-2$ mode.

A period-luminosity relationship can be deduced for the oscillations in dwarf novae, and this is in good agreement with recent calculations on quadrupole oscillations of hot white dwarfs made by Osaki and Hansen (1973).

Pulsations have so far been detected in the following objects: Z Cam (17 s), SY Cnc (24.6), CN Ori (25), KT Per (27), UX UMa (29), VW Hyi (28-34), CD - $42^{\circ} 14462$ (29 and 30), AH Her (31).
\end{abstract}

\section{References}

Osaki, Y. and Hansen, C. J.: 1973, Astrophys. J. 185, 277.

Warner, B. and Robinson, E. L.: 1972, Nature 239, 2.

Ledoux et al. (eds.), Stellar Instability and Evolution. 125, All Rights Reserved

Copyright o 1974 by the IAU. 